\title{
Screened Coulomb interactions in metallic alloys. I. Universal screening in the atomic-sphere approximation
}

\section{Ruban, Andrei; Skriver, Hans Lomholt}

\section{Published in:}

Physical Review B Condensed Matter

Link to article, DOI:

10.1103/PhysRevB.66.024201

Publication date:

2002

Document Version

Publisher's PDF, also known as Version of record

Link back to DTU Orbit

Citation (APA):

Ruban, A., \& Skriver, H. L. (2002). Screened Coulomb interactions in metallic alloys. I. Universal screening in the atomic-sphere approximation. Physical Review B Condensed Matter, 66(2), 024201.

https://doi.org/10.1103/PhysRevB.66.024201

\section{General rights}

Copyright and moral rights for the publications made accessible in the public portal are retained by the authors and/or other copyright owners and it is a condition of accessing publications that users recognise and abide by the legal requirements associated with these rights.

- Users may download and print one copy of any publication from the public portal for the purpose of private study or research.

- You may not further distribute the material or use it for any profit-making activity or commercial gain

- You may freely distribute the URL identifying the publication in the public portal 


\title{
Screened Coulomb interactions in metallic alloys. I. Universal screening in the atomic-sphere approximation
}

\author{
A. V. Ruban and H. L. Skriver \\ Center for Atomic-scale Materials Physics and Department of Physics, Technical University of Denmark, DK-2800 Lyngby, Denmark
}

(Received 18 June 2001; revised manuscript received 23 April 2002; published 26 June 2002)

\begin{abstract}
We have used the locally self-consistent Green's-function (LSGF) method in supercell calculations to establish the distribution of the net charges assigned to the atomic spheres of the alloy components in metallic alloys with different compositions and degrees of order. This allows us to determine the Madelung potential energy of a random alloy in the single-site, mean-field approximation. The Madelung potential makes densityfunctional calculations by the conventional single-site, coherent potential approximation practically identical to the more rigorous LSGF supercell results obtained with a single-site local interaction zone. We demonstrate that the basic mechanism that governs the charge distribution is the screening of the net charges of the alloy components that makes the direct Coulomb interactions short ranged. In the atomic-sphere approximation, this screening appears to be almost independent of the alloy composition, lattice spacing, and crystal structure. A formalism which allows a consistent treatment of the screened Coulomb interactions within the single-site mean-field approximation is outlined. We also derive the contribution of the screened Coulomb interactions to the $S^{(2)}$ formalism and the generalized perturbation method.
\end{abstract}

DOI: 10.1103/PhysRevB.66.024201

PACS number(s): 71.23.-k

\section{INTRODUCTION}

The coherent potential approximation (CPA), ${ }^{1-3}$ as implemented on the basis of multiple-scattering theory ${ }^{4,5}$ and combined with density-functional theory (DFT) ${ }^{6-9}$ constitutes the basis for ab inito calculations of the electronic structure and physical properties of random metallic alloys. This combination of the CPA with DFT, or, in most cases, with the local-density approximation (LDA), seems to be quite transparent ${ }^{8,9}$ leading to expressions for the one-electron potential and total energy which are very similar to those for ordered systems. However, there is, by now, a wellrecognized problem ${ }^{10-13}$ with this description related to the fact that the atomic or "muffin-tin" spheres, which artificially divide the crystal into regions associated with particular alloy components, may possess nonzero net charges.

The problem stems from the fact that the conventional single-site (SS) DFT-CPA method is based on the effectivemedium model of a random alloy which considers only conditionally averaged quantities and leads to the use of the single-site approximation not only in the electronic structure part of the problem during the solution of the CPA equations, but also in the DFT self-consistent loop in the calculations of the electrostatic contributions to the one-electron potential and energy. The single-site approximation provides no information as to the charge distribution beyond the atomic sphere of each alloy component and, since the surrounding effective medium is electroneutral, Poisson's equation cannot be solved properly if the atomic spheres have nonzero net charges. Hence to find the correct solution to Poisson's equation one must somehow describe the effect of the missing charge. Since the electron density inside each atomic sphere is well defined, any such description may be associated with a modification of the effective medium specifically for each alloy component. This may be regarded as an inconsistency since, in that case, the CPA and the electrostatic part of the DFT are based on different effective media.
One obvious solution to the problem is to use electroneutral spheres (see, for instance, Ref. 13). However, in the methods based on the atomic sphere approximation (ASA) this frequently leads to large sphere overlaps and a quite poor description of the electronic structure, especially in the case of inhomogeneous systems, such as partially ordered alloys or surfaces with an inhomogeneous concentration profile.

A more general solution can be found, however, in which the electrostatic potential is modified without making effective media for each alloy component in contradiction to the assumptions of the CPA. The way to do this is to introduce an additional shift of the one-electron potential due to the electrostatic interaction of the electrons inside each atomic sphere with the missing charge distributed outside of the sphere and postulate that the interaction comes from the boundary between the atomic sphere and the effective medium. Such a shift may be associated with an intrasite interaction, which has no connection at all to the effective medium.

This is exactly what is done in the locally self-consistent Green's-function (LSGF) method ${ }^{14}$ where one goes beyond the single-site approximation for Poisson's equation by means of a supercell which models the spatial distribution of the atoms in a random alloy while a CPA effective medium is used in the electronic structure calculations beyond a local interaction zone (LIZ). If the LIZ consists of only one atom, the LSGF method becomes equivalent to the CPA method with a properly defined electrostatic potential and energy. ${ }^{14}$ In this case, however, each atom in the supercell has its own electrostatic shift given by the Madelung potential from all the other atoms in the supercell while the effective medium is the same for all atoms. It is clear that such an additional shift for each alloy component does not interfere with the CPA because the CPA effective medium is determined on the basis of the one-electron potentials including these shifts and because the CPA itself does not impose any restriction on the one-electron potentials of the alloy components. 
Following the above arguments two groups have proposed an ad hoc expression for the electrostatic shift of the oneelectron potential due to nonzero net charges in the atomic spheres of the alloy. ${ }^{11,12,15}$ Although the basic models are seemingly different and based on different observations, either (i) the net charge of an impurity in a metal is screened beyond the first coordination shell, ${ }^{16,17}$ or (ii) the net charge of an alloy component is proportional to the number of the nearest neighbors of the opposite type,${ }^{10}$ they lead to exactly the same expression for the one-electron potential, i.e.,

$$
V_{i}=-\frac{e^{2} q_{i}}{R_{1}},
$$

where $V_{i}$ is the additional electrostatic shift of the oneelectron potential of the $i$ th alloy component of net charge $q_{i}$ and $R_{1}$ is the radius of the first coordination shell.

In fact, the models described above are practically identical to the model proposed more than three decades ago ${ }^{18,19}$ to account for charge-transfer effects in the self-consistent Hartree scheme based on the tight-binding CPA. In this scheme the variation of the $i$ th atom energy level $\delta \epsilon_{i}$ is proportional to the corresponding charge transfer $q_{i}$, i.e., $\delta \epsilon_{i}=I q_{i}$, where $I$ is some average intra-atomic Coulomb interaction. The non-self-consistent limit corresponds to $I=0$, while $I$ $=\infty$ provides local neutrality. ${ }^{19}$ In the present context one may identify $\delta \epsilon_{i}$ with $V_{i}$ and it therefore follows that $-e^{2} / R_{1}$ may be considered an intrasite Coulomb interaction.

Although there is at least some consensus concerning the definition of the additional electrostatic shift (1), which gives charge transfers quite close to the values obtained in supercell calculations, ${ }^{20}$ different workers do not agree on the corresponding electrostatic contribution to the total energy of the random alloy. Some completely deny even the possibility of having such a term in a "consistent" SS-DFT-CPA theory ${ }^{21,22}$ while others argue about the details of how this term should be defined. ${ }^{10,12,23,24}$ It would seem that the presently suggested models of charge-transfer effects in the single-site approximation to the electrostatic problem, except the trivial elimination of the net charges by adjusting the radii of the atomic spheres of the alloy components, may be considered neither exact nor even "a consistent theory." It is the main purpose of the present paper to shown that a consistent SS-DFT-CPA theory including a correct description of the charge-transfer effects does indeed exist.

Here, we define the electrostatic shift of the one-electron potential and the corresponding contribution to the total energy in a form which is very similar to that proposed by Korzhavyi et al. ${ }^{11,15}$ as well as by Johnson and Pinski ${ }^{12}$ and which provides a rigorous solution to the electrostatic problem in the single-site approximation. The actual expression for the electrostatic shift in the single-site model for Poisson's equation includes one adjustable parameter, the value of which is to be obtained in supercell calculations by the LSGF method in which the Madelung problem is solved exactly. It turns out that, as long as the ASA is used, the value of the adjustable parameter is practically independent of lattice structure, volume, and alloy composition due to the fact that the screening of the electrostatic part of the problem, in the ASA at least, is almost universal.
The paper is organized as follows. In Sec. II we outline the main concepts behind the LSGF used in this work and the details of the calculations. In Sec. III we present a pragmatic solution to the problem of finding the Madelung shift in SS-DFT calculations on the basis of the average values of the net charges and Coulomb shifts, $\left\langle q_{i}\right\rangle$ and $\left\langle V_{i}\right\rangle$, from supercell calculations. We also demonstrate that the linear relation between the net charges $q_{i}$ and the corresponding Coulomb shifts $V_{i}$ of the alloy components discovered for metallic alloys by Faulkner $e t$ al. ${ }^{25}$ is practically universal in the effective-medium approach for the Green's function. This means that the response of the electron system to the Coulomb field is linear and universal in such systems, and that the screening must be universal too. That this is indeed the case is demonstrated in Sec. IV where we calculate the distribution of the screening charge in several systems and show that it is almost independent of the crystal structure, the alloy constituents, and the composition.

In Sec. V we present a formalism for the screened Coulomb interactions in the single-site, mean-field approach for the electrostatic potential and energy and demonstrate that the conventional assumption of a vanishing Madelung potential and energy is not valid in general. Instead, one must include an additional term due to intrasite interactions which are, in fact, exactly the screened Coulomb interactions. We also discuss the ordering contribution to the Madelung energy and show why the screening contribution may be obtained in supercell calculations for ordered structures. The contribution from the screened Coulomb interaction to the generalized perturbation method and the $S^{(2)}$ formalism is also determined. Finally, in Sec. VI, we demonstrate that the total energy of a random alloy may be reproduced exactly in single-site CPA-DFT calculations with corrections due to the screening intrasite interaction.

\section{METHODOLOGY}

\section{A. Spatial ergodicity and cluster expansion}

In this paper we will consider only such alloy systems that on an underlying crystal lattice with perfect translational symmetry satisfy two conditions: (i) spatial homogeneity and (ii) no correlations between the one-electron potentials at sufficiently large distances. For the Coulomb interactions in a random alloy both conditions may be formulated explicitly in terms of the average monopole electrostatic potential $V_{i}$ in the atomic sphere around site $i$ due to the charge distribution in all of the remaining systems. In an ordered alloy this potential is the Madelung potential. Specifically, the first condition means that any real-space average values of products of potentials must be translationally invariant, i.e.,

$$
\left\langle V_{i} V_{j} \cdots V_{k}\right\rangle=\left\langle T_{a}\left(V_{i} V_{j} \cdots V_{k}\right)\right\rangle,
$$

where $T_{a}$ is the translation operator $T_{a} f(r)=f(r+a)$, and the second condition is

$$
\left\langle V_{i} V_{j} \cdots V_{k} T_{a}\left(V_{l} V_{m} \cdots V_{n}\right)\right\rangle=\left\langle V_{i} V_{j} \cdots V_{k}\right\rangle\left\langle V_{l} V_{m} \cdots V_{n}\right\rangle,
$$

for $a \rightarrow \infty$. 
According to Lifshitz et al. ${ }^{26}$ the space formed by the complete set of distinct realizations of the potential $V_{i}$ on the lattice, the operator $T_{a}$, and the property of spatial homogeneity plays the same role in the theory of disorder as the phase space, the operator of dynamical evolution, and Liouville's theorem do in statistical mechanics. Moreover, according to Birkhoff's ergodic theorem, for any functional $f\left[V_{i}\right]$, where $V_{i}$ is some random realization of the potential on the lattice we have

$$
\lim _{\Omega \rightarrow \infty} \frac{1}{\Omega} \int_{\Omega} f\left[T_{a} V_{i}\right] d \Omega=\left\langle f\left[V_{i}\right]\right\rangle,
$$

i.e., phase-space and real-space averages are equivalent. ${ }^{26}$ This equation constitutes the principle of spatial ergodicity, according to which all possible finite atomic arrangements may be realized in a single infinite sample if the conditions (2) and (3) are satisfied.

What makes the above principle work in practice is the fact that for self-averaging or "measurable" quantities which per definition have well-defined limits when the volume of the system approaches infinity all the correlations of the atomic distribution become unimportant at some distance and hence the sample may be chosen finite. This may be formulated explicitly by means of the cluster expansion theorem ${ }^{27}$ which defines the corresponding measurable quantity in terms of the site occupation correlation functions,

$$
\Pi=\pi_{0}+\sum_{f} \pi_{f} \xi_{f},
$$

where $\pi_{q}$ are the coefficients or interaction parameters, $\xi_{f}$ $=\left\langle\delta c_{i} \delta_{j} \cdots \delta c_{k}\right\rangle$ the correlation function of the figure or cluster $f$ which corresponds to a specific position of the sites $i, j$, and $k$ in the lattice, and $\delta c_{i}=c_{i}-\left\langle c_{i}\right\rangle$ is the fluctuation of the site occupation numbers $c_{i}$ taking on values 0 and 1 depending on whether site $i$ is occupied by one or the other component.

According to Eq. (5) there are two practical ways of calculating the properties of a random system for which we have $\xi_{f}=0$ and thus $\Pi_{\text {rand }}=\pi_{0}$ : (i) the cluster or supercell approach, where $\xi_{f}=0$ is satisfied on average only for those clusters $f$ for which $\pi_{f} \neq 0$, or (ii) the effective-medium approach, which directly gives $\pi_{0}$ from some knowledge of the alloy components. The first approach is realized, for instance, in the so-called special quasirandom structure (SQS) method $^{28}$ while the second approach is realized by the coherent potential approximation where the real atoms are substituted by a specifically chosen effective medium on the lattice.

\section{B. LSGF method: A combined supercell-effective-medium approach}

The supercell and the effective-medium approaches are combined into a single computational scheme in the locally self-consistent Green's-function (LSGF) method. ${ }^{14,29}$ In the LSGF method the supercell approach is used to provide the correct solution to the Madelung problem for a given alloy modeled by an appropriate supercell. It is also used in part in the electronic structure calculations, which are performed separately for every atom in the supercell by means of the local interaction zone centered at each atom. Inside the LIZ the multiple-scattering equations are solved properly, while the region outside the LIZ is represented by the effective medium, which is usually taken to be the CPA effective medium built on all the one-electron potentials in the supercell. This means that every atom of the supercell "sees" only the CPA effective medium outside the LIZ, which according to the CPA definition represents a random alloy. In other words, the one-electron Green's function of the supercell (sc) obtained in LSGF calculations may be presented as ${ }^{14,29}$

$$
G^{s c}=\sum_{i}\left[G_{i}^{0}+\sum_{f} \Delta G_{i f} \xi_{i f}^{L I Z}\right],
$$

where $G_{i}^{0}$ is the Green' function of the $i$ th atom in the supercell embedded in the CPA effective medium, $\Delta G_{i f}$ the contribution to $G_{i}^{0}$ due to the presence of the specific atomic arrangements on the figure $f$ in the LIZ as specified by the correlation function $\xi_{i f}^{L I Z}$, which is equal to that of the supercell $\xi_{i f}^{s c}$ if the figure $f$ is circumscribed by the LIZ, i.e., it can be put inside the LIZ in such a way that one of its vertices coincides with the central atom of the LIZ, otherwise $\xi_{i f}^{L I Z}$ $=0$.

Equation (6) clearly shows how the LSGF works, and in particular, how the effective-medium approach, represented by $G_{i}^{0}$, is combined with the cluster or supercell approach, represented by the second term. It follows from Eq. (6) that the LIZ allows one effectively to cut off the contributions from the clusters which are not circumscribed by the LIZ (an equivalent formulation in terms of effective interactions is given in Ref. 29). If the LIZ is single site, i.e., it consists of only one atom $(\mathrm{LIZ}=1)$, the contributions from the second term in Eq. (6) vanish and we are left with the usual CPA or pure effective-medium approach to the electronic structure problem. It is this single-site approach, referred to as SSLSGF, which will be used in most of the present paper. It has the advantage over the usual SS-DFT-CPA method that Poisson's equation is solved exactly within a given approximation for the form of the electron density.

Note, however, that the LSGF method is by no means restricted to the single-site approximation. In fact, it allows us to include local environment effects in the electronic structure calculations for the figures circumscribed by the LIZ if on average $\xi_{f}^{\langle L I Z\rangle}=\xi_{f}^{s c}=0$ which is the case in a random alloy. In this respect the LSGF method may be considered a self-consistent embedded-cluster method (ECM) of the kind proposed by Gonis et al. ${ }^{30}$ more than two decades ago. With a proper choice of the supercell used to model a given random alloy ${ }^{29}$ the LSGF solves two major problems of the ECM: (i) it provides a set of clusters to represent an alloy with a given short-range order and (ii) it allows one to close the DFT loop with the correct treatment of the electrostatics.

There is one important point concerning the electronic structure obtained in the LSGF with the CPA effective medium which should be mentioned: Despite the fact that it can be quantitatively accurate, it is qualitatively different from 
the electronic structure which would result from direct supercell calculations with periodic boundary conditions. That is, the electronic spectrum in the LSGF-CPA method is always complex, unless all the atoms are equivalent in the supercell (pure metal) or the size of the LIZ is infinite. Thus in the LSGF-CPA the electronic structure of an ordered alloy is never correct, although it may be calculated with arbitrarily high accuracy. On the other hand, since Blochs' theorem is not applied to the supercell during the electronic structure calculations, the LSGF method is a perfect tool for calculating SQS as opposed to the ordinary band-structure methods, which in this case lead to a real, i.e., qualitatively incorrect, electronic structure of the random alloys.

\section{Choice of the supercell in the LSGF and details of the calculations}

It is possible to obtain the Madelung potential and energy by a combined supercell (cluster) and effective-medium approach similar to that used in the Green's-function approach to the electronic structure problem within the LSGF method. However, this requires some knowledge of the charge-charge correlations or the screening in the alloy. Hence the only way to solve the problem is to use a supercell model with the Madelung potential and energy determined exactly from the bare electrostatic interactions, as it is usually done. Here, another problem arises: The supercell should be constructed such as to provide zero correlation functions up to the distance where the net charges of the alloy components become uncorrelated or completely screened.

In the calculations presented below we assume at the outset the existence of a short-range screening which occurs over the distance of the first several coordination shells. This assumption is based on results obtained by the chargecorrelated model, ${ }^{10}$ on single-impurity calculations, ${ }^{16,17}$ as well as on the most recent LSGF calculations by Ujfalussy et $a .^{22}$ The latter authors demonstrated that a 16-atom supercell for an fcc equiatomic random alloy, in which the SRO parameter at the eighth coordination shell must be equal to 1 due to the translation symmetry, i.e., all the atoms in the eighth coordination shell are the same as that at the central site, yields practically the same average charge transfer and total energy as a 250-atom supercell, in which the SRO parameter at the eighth coordination shell corresponding to a random number generator distribution of the alloy components on the lattice should be $\ll 1$ (see also Ref. 31 ).

Thus in all the random alloys considered below the distribution of the atoms in the supercell was chosen such that the SRO parameters (or pair-correlation functions, $\xi_{f}^{(2)}$ ) were exactly zero at least in the first six coordination shells and small (not greater than 0.01 in absolute value) up to the eighth coordination shell. Although the multisite correlation functions have not been optimized, they should not play a significant role in the ASA where only monopole intersite Coulomb interactions are taken into account.

The electronic structure calculations were in all cases performed in the scalar relativistic approximation by the Korringa-Kohn-Rostoker (KKR)-ASA technique ${ }^{32}$ with an $s$, $p$, and $d$ basis in the framework of either the usual SS-DFT-
CPA or the LSGF methods with a CPA effective-medium. The ASA (no multipole corrections to the Madelung potential and energy) has been used in the electrostatic part of the problem. The integration of the Green's function over energy was performed in the complex plane over 16-20 energy points on a semicircular contour. The local-density approximation was used in the DFT part with the Perdew and Zunger $^{33}$ parametrization of the results by Ceperly and Alder. $^{34}$

\section{NET CHARGE AND MADELUNG POTENTIAL IN METALLIC ALLOYS}

Here we discuss a pragmatic solution to the following problem: Can one devise a Madelung potential for the alloy components to be used in SS-DFT-CPA calculations such that the charge-transfer effects, i.e., the net charges of the alloy components, are consistent with those obtained in SSLSGF calculations where charge-transfer effects are treated properly? The fact that such a potential can be found may seem surprising in view of the principal differences between the LSGF and the SS-DFT-CPA methods. In the LSGF approach all the atoms in the supercell are different due to their different local environment while in the usual SS-DFTCPA approach one deals only with average quantities, i.e., in the terminology of Ref. 35, the LSGF supercell approach is equivalent to the polymorphous model of the alloy while the effective-medium approach is equivalent to the isomorphous model. However, it is obvious that this can be done on average.

It was discovered by Faulkner et al. $^{25}$ from supercell calculations that the net charges on different sites $i, q_{i}$, and the corresponding Madelung potentials $V_{i}$ obey a linear relationship. In Fig. 1 we show such a $q V$ relation for a 512-atom supercell which models a random $\mathrm{Al}_{50} \mathrm{Li}_{50}$ alloy on an underlying fcc lattice. For comparison we also show the charge and Madelung shift for AlLi in the ordered $L 1_{0}$ structure. All results are obtained by LSGF calculations with the CPA effective medium in (i) the single-site approximation for the electronic part of the problem, SS-LSGF, i.e., LIZ=1, (upper panel) and (ii) with the perturbation in the electronic structure caused by the local environment up to the second coordination shell, i.e., embedded cluster (EC)-LSGF, LIZ=3 (lower panel), included in the Green's function. For random alloys the inclusion of more distant coordination shells do not affect the results significantly and thus the $L I Z=3$ results may be considered to be converged in the LIZ size.

The most striking feature of the $q V$ relation obtained in the SS-LSGF calculations is the perfect alignment of the $q V$ points along two almost straight lines, one for each alloy component. This is, in fact, very similar to what has been observed by Pinski $^{36}$ in model calculations using the Thomas-Fermi approximation. Furthermore, a change of the ratio of the atomic sphere radii of the alloy components, $r$ $=S_{A l} / S_{L i}$, leads to a rescaling of the $q V$ points. Hence for a specific ratio, $r=1.12$ in the present case, the $q V$ relation collapses into the single point: $(q, V)=(0,0)$. The existence of this point in the SS-LSGF is a consequence of the fact that all $\mathrm{Al}$ atoms as well as all $\mathrm{Li}$ atoms become indistinguishable 


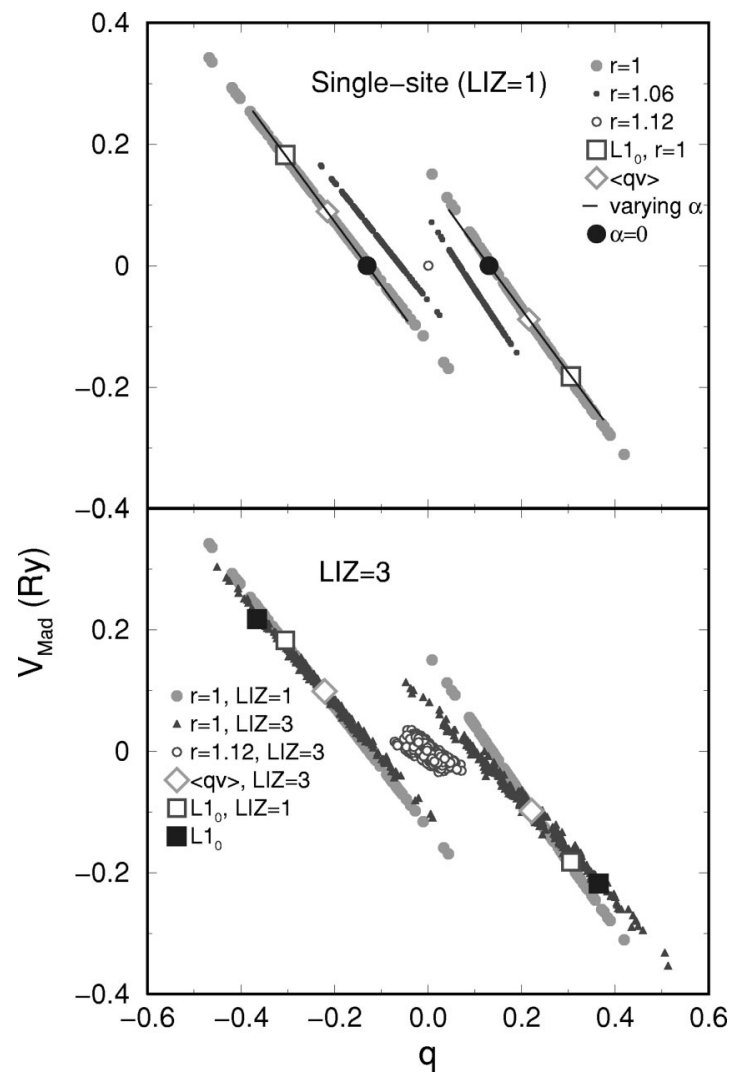

FIG. 1. The distribution of the net charges $q_{i}$ and corresponding Madelung potentials $V_{i}$ in the 512-atom supercell, modeling a random $\mathrm{Al}_{50} \mathrm{Li}_{50}$ alloy, ordered $L 1_{0}$ alloy, and in the single-site CPADFT calculations obtained by varying $\alpha$.

if the net charges of the alloy components are zero: The difference between the atoms caused by local environment effects is solely due to the Madelung shift, which is zero in this case. Thus for this particular choice of $r$ the polymorphous model is identical to the usual isomorphous model, or the SS-LSGF method is identical to the SS-DFT-CPA method.

On the other hand, it is clear that the two models are not equivalent when local environment effects are included in the electronic structure part of the LSGF method, i.e, for LIZ $>1$. This is demonstrated in the lower panel of Fig. 1 where the local environment effects are clearly seen to destroy the strict alignment of the $q V$ points and, as consequence, the possibility of choosing electroneutral atomic spheres by a single $r$ value. However, even if this were possible, all the atoms, or the corresponding one-electron potentials, would still be different.

The discussion of local environment effects is beyond the scope of the present paper, and the results are included only to demonstrate the qualitative difference between the correct results and those obtained by the LSGF method in the singlesite approximation: Inclusion of intersite correlations in the electronic structure calculations leads to a real polymorphous description of random alloys which cannot be mimicked by a single-site LIZ. As a consequence, as we will show later the SS-DFT-CPA method can reproduce the results of the SSLSGF exactly, but will, in general, reproduce only approxi- mately the correct solution to the supercell or polymorphous model of a random alloy.

The two $q V$ points for the ordered $L 1_{0}$ structure are seen to fall on the $q V$ lines for the random alloy as already noted in Ref. 25), and, in fact, all the points on the $q V$ relation obtained by the SS-LSGF method may be reproduced by a series of ordinary SS-DFT-CPA calculations, by using the shift of the one-electron potential defined in a way similar to Eq. (1), i.e.,

$$
V_{i}=-\alpha \frac{e^{2} q_{i}}{S}
$$

where $q_{i}$ is the net charge of the alloy components, $S$ the Wigner-Seitz radius, and $\alpha$ a parameter which may be varied arbitrarily in the SS-DFT-CPA calculations without specifying its physical meaning. However, it is important to note that $\alpha=-\infty$ corresponds to the electroneutral case $\left(q_{i}=0\right)$ and $\alpha=0$ to the limit where there is no response of the system to charge-transfer effects. As we will see later, the values of the net charges $q_{0 i}$ obtained in the SS-DFT-CPA calculations with $\alpha=0$ are important scaling parameters. It is also useful to note that for the $L 1_{0}$ structure $\alpha_{L 1_{0}}$ $=0.8811575,{ }^{37}$ and in the screened impurity model (1) $\alpha_{S I M}=0.552669$ and 0.568542 for the fcc and bec crystal structures, respectively.

Figure 1 shows the $q V$ relation, indicated by the black line, obtained in the SS-DFT-CPA calculations including Eq. (7) with $\alpha$ varying from -1.5 to 5 together with the SSLSGF results, gray circles. It is clearly seen that the isomorphous and polymorphous $q V$ relations coincide, and this allows one to make an isomorphous model consistent with the polymorphous results. The point is that all the net charges and corresponding Coulomb shifts in the polymorphous model have significance only in terms of the average values they produce. This is so, because every supercell has its own set of net charges and Madelung shifts and, in the case of an infinite system, there is an infinite number of different $q V$ points. Their average values, $\left\langle q_{i}\right\rangle$ and $\left\langle V_{i}\right\rangle$, however, have a well-defined physical meaning as conditional averages of self-averaging quantities, and thus it is the average $\langle q V\rangle$ point which must be reproduced by the isomorphous model. Hence for a random alloy $\alpha$ is given by

$$
\alpha_{\text {rand }}=-\frac{S}{e^{2}} \frac{\left\langle V_{i}\right\rangle}{\left\langle q_{i}\right\rangle} .
$$

Note that in a binary AB alloy, it clearly does not matter, for which alloy component $i=A, B, \alpha_{\text {rand }}$ is determined, since $\left\langle V_{A}\right\rangle /\left\langle q_{A}\right\rangle=\left\langle V_{B}\right\rangle /\left\langle q_{B}\right\rangle .{ }^{25}$ The same is true for multicomponent alloys, but in this case, rather than being a trivial consequence of the charge neutrality condition, it follows from the physical origin of $\alpha_{\text {rand }}$, which will be discussed in the next two sections. For an fcc $\mathrm{Al}_{50} \mathrm{Li}_{50}$ alloy at $S=2.954$ a.u. we find from the LSGF calculations $\alpha_{\text {rand }}=0.60716$.

Of course, the coincidence of $\left(\left\langle q_{i}\right\rangle,\left\langle V_{i}\right\rangle\right)$ is a necessary but not sufficient condition for the equivalence of the isomorphous and polymorphous models. The two models may be called equivalent only if the electronic structure of the 


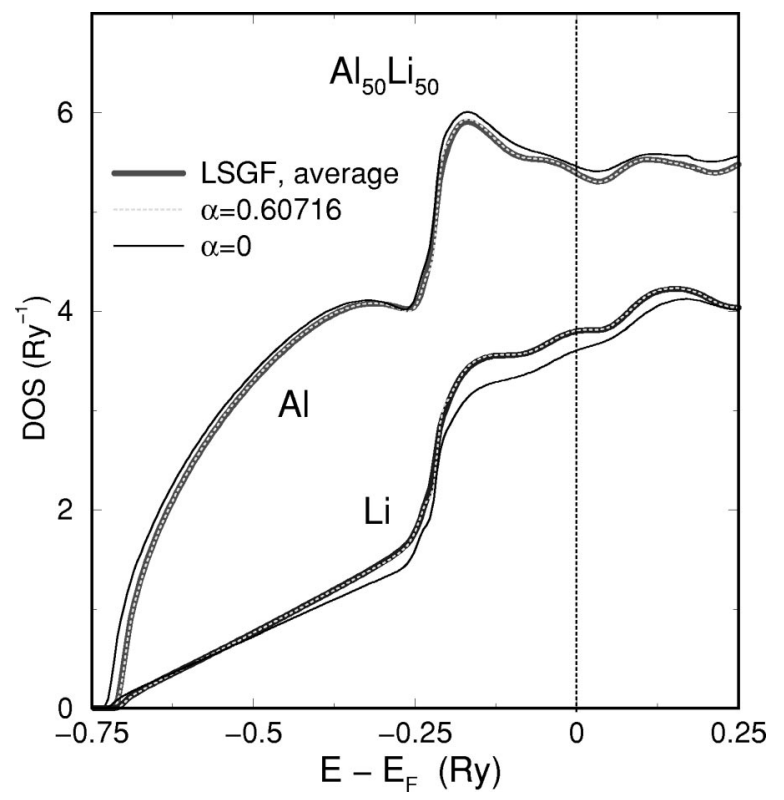

FIG. 2. The local density of states in the $\mathrm{Al}_{50} \mathrm{Li}_{50}$ obtained by the LSGF method with LIZ=1 and by the single-site CPA-DFT with different values of $\alpha_{\text {rand }}$.

random alloy and its conditional averages agree. In Fig. 2 we show that this is indeed the case: The local densities of states (DOS) for the $\mathrm{Al}$ and $\mathrm{Li}$ atoms in $\mathrm{Al}_{50} \mathrm{Li}_{50}$ calculated by the SS-DFT-CPA method coincide with the corresponding conditional average state densities obtained in the SS-LSGF calculations for the 512-atom supercell. For comparison we also show the DOS obtained with $\alpha=0$ corresponding to the "conventional" CPA. Although the latter differs from the correct state density, it is obvious that neglecting the electrostatic shift (7) has only a minor effect on the DOS.

The reason why the average state densities coincide is the following: In the SS-LSGF method the difference between the atoms of the same type comes only through the corresponding Madelung shift. A shift in potential leads to a change in the charge transfer through a skewing of the local DOS as seen in Fig. 3. Therefore, when the conditionally averaged DOS is obtained, the skewing contributions from the individual atomic sites caused by $V_{i}$ will cancel and leave only the DOS given by the average $\left\langle V_{i}\right\rangle$. Of course, this is true only in the SS-LSGF method (LIZ=1). In fact, the local environment effects in concentrated random alloys may influence quite strongly the electronic structure of the central site of the LIZ.

To investigate how the $q V$ relation depends on the system we show in Fig. $4 q V$ relations for five different systems including a $\mathrm{Cu}$ impurity in $\mathrm{Pt}(S=3$ a.u.) and four random alloys: fcc $\mathrm{Cu}_{50} \mathrm{Pt}_{50}\left(S=3\right.$ a.u.), fcc $\mathrm{Al}_{50} \mathrm{Li}_{50}(S=2.954$ a.u. $)$, bcc $\mathrm{Cu}_{50} \mathrm{Zn}_{50}\left(S=2.7\right.$ a.u.), and ternary fcc $\mathrm{Cu}_{50} \mathrm{Ni}_{25} \mathrm{Zn}_{50}$ ( $S=2.65$ a.u.). In the plot all charges have been normalized by $q_{0 i}$ obtained in the no response limit, i.e., $\alpha=0$ or $V_{i}$ $=0$, and all Madelung shifts have been normalized by $q_{0 i} / S$. To partly simplify the plot we have used $\left|q_{0 i}\right|$ in the normalizations, thereby separating the $q V$ relations into two lines rather than one.

The results presented in Fig. 4 show the existence of a

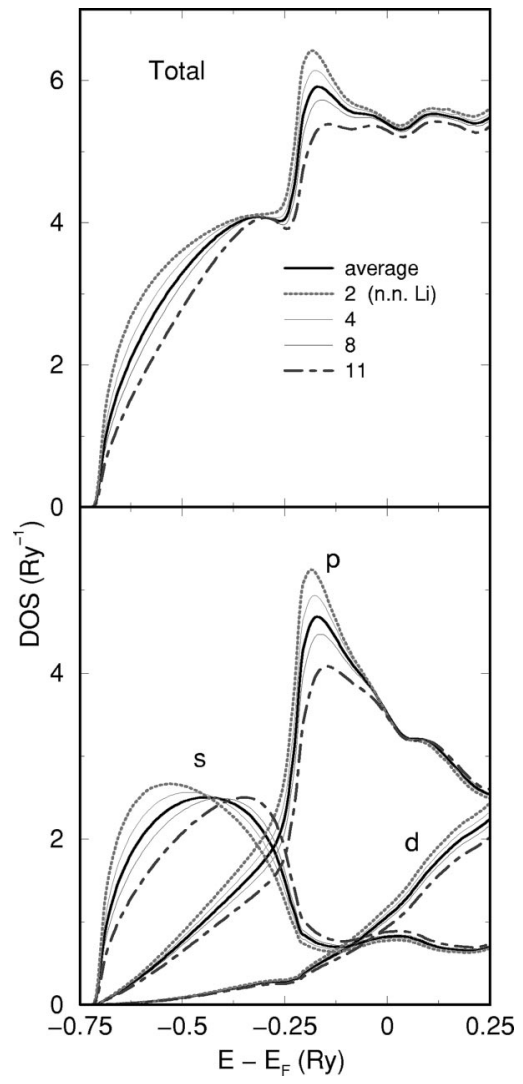

FIG. 3. The site- and $l$-projected density of states for $\mathrm{Al}$ atoms in $\mathrm{Al}_{50} \mathrm{Li}_{50}$ having different Madeulng potentials due to different numbers of nearest-neighbor $\mathrm{Li}$ atoms.

universal $q V$ relation. Or in other words, the linear-response function $\chi$ which gives the change in the net charge relative to $q_{0 i}$ caused by $V_{i}$, i.e., $q_{i}-q_{0 i}=1 / e^{2} \chi V_{i} S$, is a universal constant in metallic alloys in the ASA. From the results presented in the figure we find that $\chi \approx-0.63$. This unavoidably leads to the existence of a single, unique $\alpha_{\text {rand }}$ as witnessed by the coincidence of all the $\langle q V\rangle$ points in Fig. 4. Strictly speaking, the slopes of the $q V$ lines are not exactly identical and, in fact, $\alpha_{\text {rand }}$ varies from 0.6 in $\mathrm{Cu}_{50} \mathrm{Zn}_{50}$ and $\mathrm{Cu}_{50} \mathrm{Ni}_{25} \mathrm{Zn}_{50}$ to 0.615 in LiMg alloys, not included in the figure. However, for most practical purposes the choice $\alpha_{\text {rand }}=0.607$ provides a sufficiently accurate description of the electronic structure of random alloys in the SS-DFT-CPA method in the ASA for the electrostatic part.

\section{SCREENING CHARGE IN METALLIC ALLOYS}

In the previous section we have, in effect, defined a procedure whereby SS-DFT-CPA calculations may provide a rigorous solution to the electrostatic problem in random alloys. The only requirements are that the Madelung shift (7) is included and that the constant $\alpha_{\text {rand }}$ is obtained from Eq. (8) with the average Madelung potential and net charges of the alloy components determined in supercell calculations by the SS-LSGF method. In the derivation of the procedure we have used some general arguments which do not clarify the physical origin of the universal value of $\alpha_{\text {rand }}$. However, it 


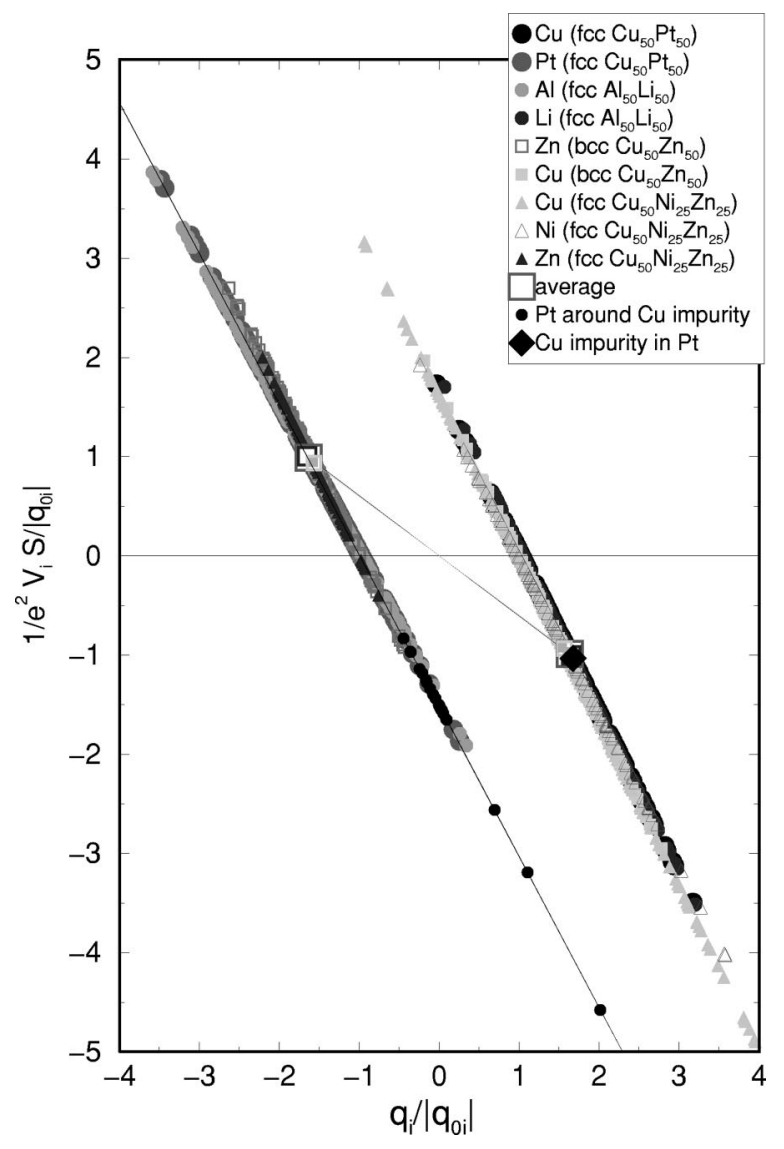

FIG. 4. $q V$ relation scaled by $q_{0 i}$, and the Wigner-Seits radius $S$.

is clear that Eq. (7) accounts for the missing charge $-q_{i}$ in the single-site Poisson equation for the $i$ th atomic sphere. Thus $\alpha_{\text {rand }}$ must be connected to the screening.

The linear character of the $q V$ relation indicates that the screening in the impurity case as well as in the case of a random alloy may be very well described by linear-response theory. Owing to enhanced electron scattering at opposite regions of the Fermi-surface linear response predicts in the case of a free-electron gas the existence of long-range Friedel oscillations, which, however, decrease relatively fast $\left(\sim r^{-3}\right)$ with the distance. In a random alloy, on the other hand, the screening is much more efficient due to the finite lifetime of the Bloch states for the underlying crystal lattice and the spatial distribution of the screening density decays exponentially. In this respect the charge correlated (cc) model $^{10,31}$ adopted by Johnson and Pinski ${ }^{12}$ in the cc- and screened-CPA method or the equivalent screened impurity model ${ }^{11,23,24}$ may be viewed as the first approximations to a description of the screening.

Based on the fact that a single impurity in a metallic host is a particular case of a dilute random alloy, one would expect, and the results for a single $\mathrm{Cu}$ impurity in $\mathrm{Pt}$ presented in the previous section unambiguously indicate this, that the screening effects in the two cases are similar. It is therefore surprising that Faulkner et al. ${ }^{35}$ and Ujfalussy et al. ${ }^{22}$ claim that the screening in a random alloy is qualitatively different from that found in a single impurity system. In fact, these authors found extremely long-range correlations between the Madelung potential at some particular site and the net charges at the other sites. Since the Madelung shift on a site is proportional to the net charge on the site, this may happen only if there are extremely long-ranged correlations between net charges or, in other words, there is no screening. However, these results were obtained on the basis of summations of the direct or bare Coulomb interactions which is, at best, an ill-defined procedure, even mathematically.

To clarify the issue of screening we will perform the following computer experiment which will allow us to establish the range of the net-charge correlations or the screening in random alloys for one particular site. We set up a 512-atom supercell which represents an fcc $\mathrm{Cu}_{50} \mathrm{Pt}_{50}$ random alloy (all SRO parameters are equal to zero up to the sixth coordination shell and $\sim 0$ for at least the next ten coordination shells) and perform self-consistent SS-LSGF $(\mathrm{LIZ}=1)$ and ECLSGF ( $\mathrm{LIZ}=3$ ) calculations. We then substitute one Pt atom with one $\mathrm{Cu}$ atom in some site which, in general, may be chosen arbitrarily. However, to keep the atomic distribution as close as possible to the random distribution we chose a site the local environment of which corresponds to the random alloy (having equal number of $\mathrm{Cu}$ and $\mathrm{Pt}$ atoms) for the first three coordination shells. We then repeat the selfconsistent LSGF calculations for the supercell with the substituted atom and find new values for the net charges in the supercell. It is clear that, when $\mathrm{LIZ}=1$, the difference between the net charges in the two calculations, $\Delta q_{i}$, gives the charges induced by the change of the net charge at the substitution site (in the case of $\mathrm{LIZ}=3$ the local environment effects also effect the charge transfer). This charge is simply the screening charge.

In the upper panel of Fig. 5 we have plotted the normalized, induced charges,

$$
Q_{i}=\frac{\Delta q_{i}}{\Delta q_{0}},
$$

at the first eight coordination shells around the substitution site $i=0$ for $\mathrm{Cu}-\mathrm{Pt}$ substitution in $\mathrm{C}_{50} \mathrm{Pt}_{50}$ random alloy and for $\mathrm{Cu}-\mathrm{Pt}$ substitution in pure $\mathrm{Pt}(S=3$ a.u.) obtained in the single-site approximation for the electronic structure $(\mathrm{LIZ}=1)$ as well as with local environment effects included $(L I Z=3)$. One may see that, while the local environment in a pure metal hardly affects the distribution of the net charges, it does introduce a dispersion in the distribution of the net charges in the random alloy, which is quite substantial at the first coordination shell but which practically disappears beyond the fifth coordination shell where, in fact, all the induced charges almost vanish.

To demonstrate that the net charge of the $\mathrm{Cu}$ "impurity" indeed becomes screened we show in the lower panel of Fig. 5 the total normalized induced charge in the $i$ th shell,

$$
Q_{\text {tot }}^{i}=\sum_{j=0}^{i} z_{j} Q_{j},
$$

where $z_{j}$ is the coordination number of the $j$ th shell. It is seen that $Q_{i}$ vanishes beyond the seventh coordination shell 


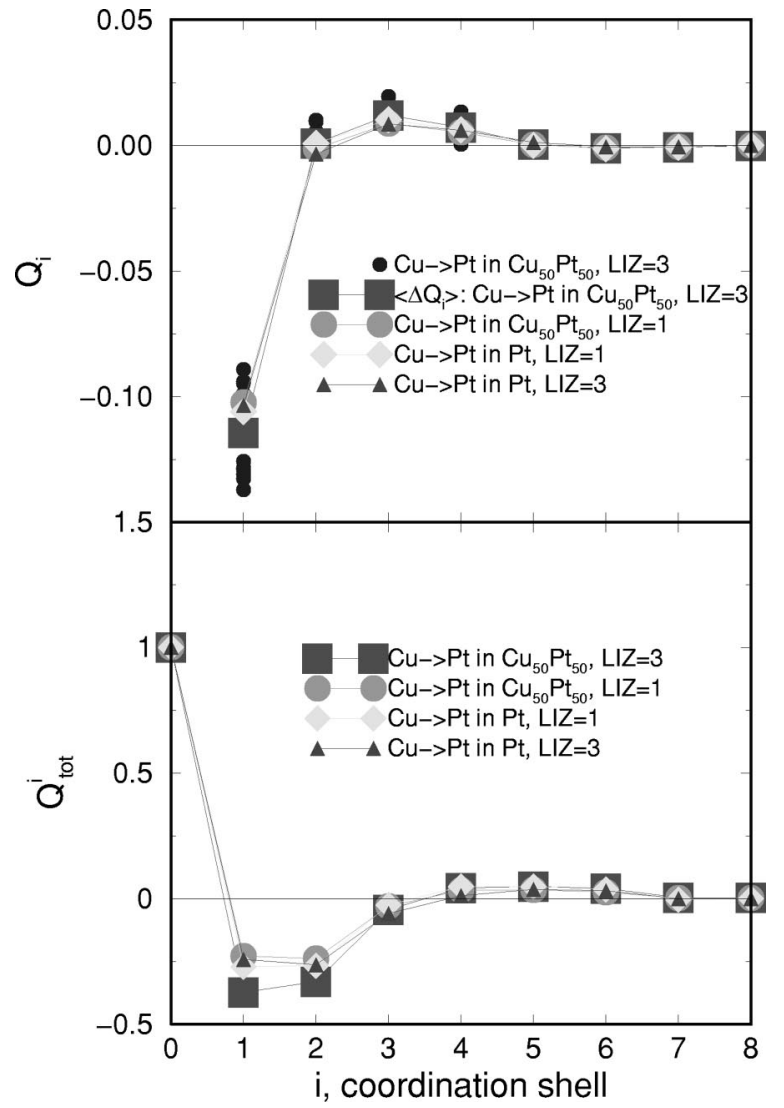

FIG. 5. The distribution of the screening charge in the random $\mathrm{Cu}_{50} \mathrm{Pt}_{50}$ alloy and in $\mathrm{Pt}$ due to substitution of a $\mathrm{Cu}$ atom.

in all cases, and we conclude that the screening in a random alloy in the single-site approximation is practically the same as the screening in the case of a single impurity in a pure metal. There are neither qualitative nor quantitative differences between the impurity and the alloy cases.

In the upper panel of Fig. 6 we show the distribution of the screening charge (not to be confused with the screening density: the screening charge is, in fact, the screening density integrated in the corresponding atomic sphere) for a $\mathrm{Cu}$ impurity in fcc, bcc, and bct Pt plotted as a function of the distance from the impurity site in units of the Wigner-Seits radius $S$. It is clear that the screening charge follows a single, common curve which does not depend on the structure. In fact, by changing the $c / a$ ratio in the bct structure one may completely fill the remaining gaps in the calculated curve. In the lower panel of the figure we have collected the results for the distribution of the screening charges in seven different systems including such hosts as Pt, $\mathrm{Al}, \mathrm{Cu}, \mathrm{V}, \mathrm{Na}$, and $\mathrm{K}$. It appears that the screening in metallic alloys depends neither on the crystal structure nor on the nature of the alloy components, at least, when described within the ASA.

The universal picture of the screening in alloy systems found above is partly destroyed when the electrostatics is treated more correctly, for instance by including multipole moment contributions to the one electron Madelung potential and energy. However, the ASA still gives a qualitatively correct picture and catches the main physics behind the phe-

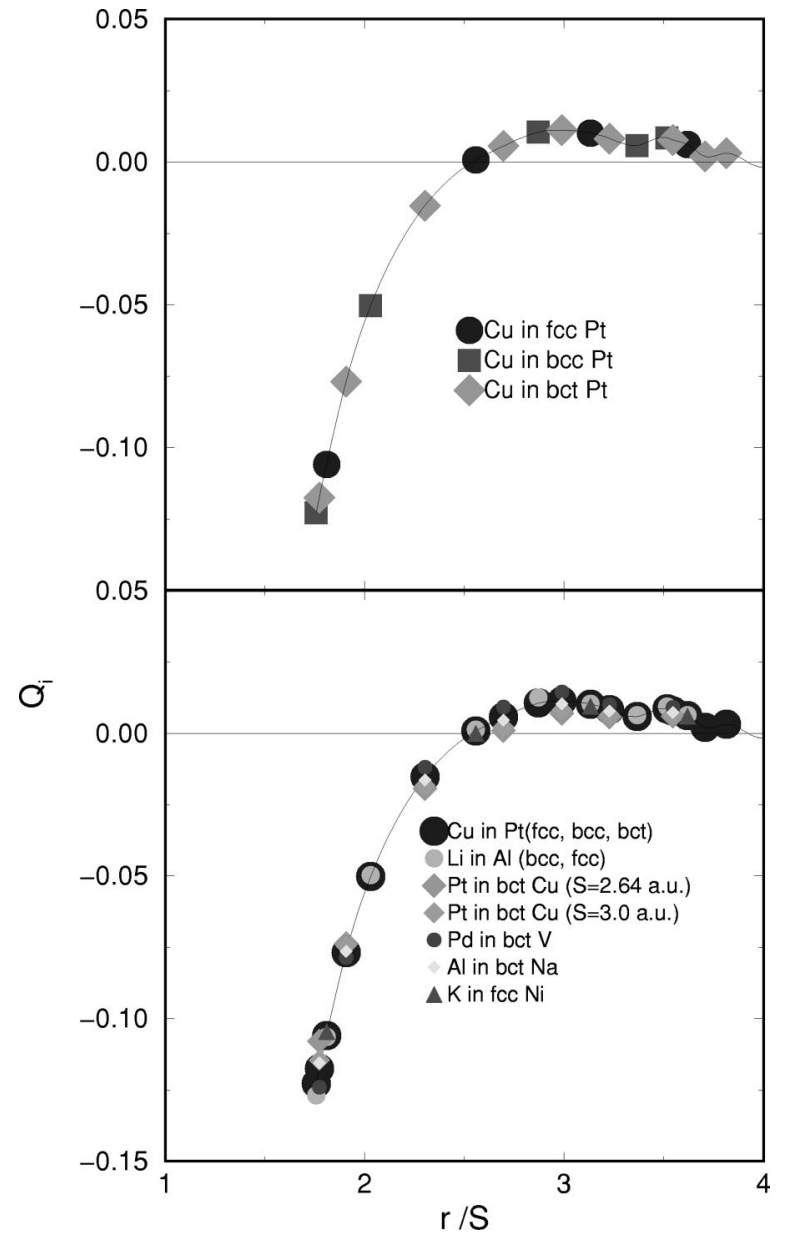

FIG. 6. The distribution of the screening charge in different metals having different crystal structure and lattice parameter.

nomenon. Hence it is worthwhile to discuss the origin of such a universality in both the screening and the response function.

First of all, it was understood a long time ago that the net charges in the atomic spheres of the alloy components have very little in common with the charger transfer in terms of the redistribution of the electron charge between the alloy components (see, for instance Ref. 38). Even in the case of the so-called ionic solids the self-consistent charge distribution is very close to that obtained from a linear superposition of the free-atom electron-densities ${ }^{39}$ and this is the reason for the success of the charge-correlated model, ${ }^{10}$ in which the net charge is proportional to the number of nearest neighbors of the opposite type (see also Ref. 31).

What we are seeing is basically a size effect: The net charges originate from the redistribution of the electron density in the interstitial region between the atomic spheres. The electron density in metals and their alloys in this region is very smooth and may be well described by a free-electron model, even for transition-metal alloys. The interstitial density is much easier to perturb than the density closer to the atomic nuclei and it participates in the screening. On this basis one may, in fact, develop a model based on linearresponse theory which leads to a semianalytical description of the universal screening. However, this is beyond the scope of the present paper. 
The calculated distribution of the screening net charge may be used to obtain the screening contribution to the oneelectron potential in the single-site model for the Poisson equation given by Eq. (7) with $\alpha$ equal to

$$
\alpha_{s c r}=\frac{S}{e^{2}} \sum_{i} z_{i} \frac{Q_{i}}{R_{i}},
$$

where $R_{i}$ is the radius of the $i$ th shell with coordination number $z_{i}$. Using the results for the screening charge $Q_{i}$ in the case of $\mathrm{Cu}-\mathrm{Pt}$ substitution for one particular site (not on average) in the $\mathrm{Cu}_{50} \mathrm{Pt}_{50}$ random alloy we find from Eq. (11) after summation up to the eighth coordination shell that $\alpha_{s c r}=0.60572$. At the same time, the average values of the net charges and the Madelung potentials, $\langle q\rangle$ and $\langle V\rangle$, in conjunction with Eq. (8) gives $\alpha_{\text {rand }}=0.60530$. That is, $\alpha_{\text {rand }}=\alpha_{s c r}$. Since there is only one effective medium in the supercell SS-LSGF calculations, it is obvious that the screening is the same for all the sites and thus should not depend on the alloy component, that is, the ratio $\left\langle V_{i}\right\rangle /\left\langle q_{i}\right\rangle$ does not depend on the alloy components.

The important point here is the fact that, since $\alpha_{s c r}$ is given by the electrostatic interactions of the net charge inside a sphere with its own screening (or missing) charge, it has an on-site or intrasite nature. Such an intrasite screening interaction depends almost entirely on the type of the system or effective medium and very little on the structure. In contrast, the parameter $\alpha_{L 1_{0}}$ (see the previous section) which allows one to perform SS-DFT-CPA calculations identical to those of the SS-LSGF for the $L 1_{0}$ structure depends solely on the geometry of the $L 1_{0}$ structure.

That is, there is no connection between the Madelung constant for an ordered structure and $\alpha_{s c r}$ : The former describes the bare electrostatic interactions between the net charges on different sites, while the latter arises due to the screening in the system and describes the real response of the electronic subsystem to the perturbation, which "dresses" the intersite Coulomb interactions making them short range. The reason why it is possible to obtain $\alpha_{s c r}$ from supercell calculations for the completely ordered structures is the fact that at the large distances, where the atomic-distribution correlation functions are not zero anymore due to the translational symmetry of the supercell, the real net charges have become screened or uncorrelated. We will return to this point in the next section, but here we would like to comment on the use of the single-site approximation in the Green's function calculations.

Our LSGF calculations of impurities in different metals indicate that the problems observed in the single-site Green's function impurity calculations by Stefanou ${ }^{17}$ and Drittler et al $^{40}$ do not originate from the single-site approximation for the Dyson equation, but from Poisson's equation, which these authors also solve in the single-site approximation. The use of the screening electrostatic shift for the one-electron potential allows one to solve the impurity problem in the single-site Green's function formalism in the ASA or in the muffin-tin (MT) approximation almost exactly. This is so because the impurity case corresponds to the dilute limit of a random alloy where the concentration of one of the alloy components approaches zero. In this case, the contribution to the electronic structure due to the local environment effects becomes negligible, and the electronic structure of the impurity obtained by the single-site or the cluster Dyson equations become almost identical. Such an effect may be seen, for instance, in Fig. 5: The dispersion of the screening net charge found in the concentrated alloy case, i.e., $\mathrm{Cu}_{50} \mathrm{Pt}_{50}(\mathrm{LIZ}=3)$, vanishes in the case of a $\mathrm{Cu}$ impurity in Pt. The effect is, in fact, the origin of the increasing accuracy of the CPA with decreasing concentration of one of the alloy components.

\section{FORMALISM FOR THE SCREENED COULOMB INTERACTIONS}

\section{A. Madelung energy of a random alloy in the single-site mean-field approximation (effective-medium approach)}

The existence of on-site or intrasite interactions of the kind discussed in connection with Eq. (11) must lead to a modified description of the electrostatics of random alloys in the single-site mean-field approximation, where all the $A$ and all the $B$ atoms are represented only by the appropriate conditional averages. It has been shown by Krasko ${ }^{41}$ that in a system with randomly distributed $A$ and $B$ ions of charge $Z_{A}$ and $Z_{B}$, respectively, embedded in a medium of compensating charges, the electrostatic energy is

$$
E_{\text {Mad }}^{r a n d-s s}=\frac{e^{2}}{2} \frac{\alpha_{M}}{S} \widetilde{Z}^{2},
$$

where $\alpha_{M}$ is the Madelung constant of the underlying lattice, $S$ the radius of the Wigner-Seitz sphere, and $\widetilde{Z}$ the average charge equal to $c Z_{A}+(1-c) Z_{B}$.

It is obvious that Eq. (12) is valid also in the CPA-DFT if one substitutes the ion charges $Z_{i}$ by the net charges $q_{i}$ of the atomic spheres. In the ASA the average charge $c q_{A}+(1$ $-c) q_{B}$ is zero, and in this particular approximation the Madelung energy vanishes. Note, however, that it vanishes neither in inhomogeneous systems ${ }^{24,32}$ nor in the MT approximation. $^{9}$

We will now reformulate the above description by including the on-site screened Coulomb interactions. However, instead of simply adding the appropriate on-site term to Eq. (12), we will use concentration wave theory to clarify the effective-medium approach to the Madelung energy in ordered alloys. Assuming that only on-site and pairwise interactions are important the Hamiltonian of a binary $A_{c} B_{1-c}$ alloy may be written as

$$
\begin{aligned}
H= & \sum_{R}\left[\epsilon_{0}^{A} c_{R}+\epsilon_{0}^{B}\left(1-c_{R}\right)\right] \\
& +\frac{1}{2} \sum_{R \neq R^{\prime}}\left[v_{R R^{\prime}}^{A A} c_{R} c_{R^{\prime}}+v_{R R^{\prime}}^{A B}\left(1-c_{R}\right) c_{R^{\prime}}\right. \\
& \left.+v_{R R^{\prime}}^{A B} c_{R}\left(1-c_{R^{\prime}}\right)+v_{R R^{\prime}}^{B B}\left(1-c_{R}\right)\left(1-c_{R^{\prime}}\right)\right],
\end{aligned}
$$

where $\epsilon_{0}^{X}$ are on-site or intrasite interactions, which we will assume depend only on the type of atom on site $R, v_{R R^{\prime}}^{X Y}$ are pair potentials acting between $X$ and $Y$ atoms at site $R$ and 
$R^{\prime}$, respectively, and $c_{R}$ is the site-occupation operator taking on the value 1 if there is an $A$ atom on site $R$ and 0 otherwise. Using $\delta c_{R}$, defined by $c_{R}=c+\delta c_{R}$, we may rewrite the Hamiltonian in the equivalent form

$$
\begin{aligned}
H= & \frac{1}{2} \sum_{R, R^{\prime}} V_{R R^{\prime}} \delta c_{R} \delta c_{R^{\prime}} \\
& +\frac{1}{2} \sum_{R \neq R^{\prime}}\left[c^{2} v_{R R^{\prime}}^{A B}+2 c(1-c) v_{R R^{\prime}}^{A B}+(1-c)^{2} v_{R R^{\prime}}^{B B}\right],
\end{aligned}
$$

where the first term includes the intrasite interaction $(R$ $\left.=R^{\prime}\right)$,

$$
V_{R=0}=2\left(\frac{1}{(1-c)} \epsilon_{0}^{A}+\frac{1}{c} \epsilon_{0}^{B}\right)
$$

as well as the intersite interactions $\left(R \neq R^{\prime}\right)$,

$$
V_{R R^{\prime}}=v_{R R^{\prime}}^{A A}+v_{R R^{\prime}}^{B B}-2 v_{R R^{\prime}}^{A B} .
$$

Upon Fourier transformation of the first term we find

$$
\begin{aligned}
H= & \frac{N}{2 \Omega_{B Z}} \int_{B Z} d q V(q) c_{q} c_{q}^{*} \\
& +\frac{1}{2} \sum_{R \neq R^{\prime}}\left[c^{2} v_{R R^{\prime}}^{A B}+2 c(1-c) v_{R R^{\prime}}^{A B}+(1-c)^{2} v_{R R^{\prime}}^{B B}\right],
\end{aligned}
$$

where the second term is the average contribution to the energy due to pair interactions which in the case of direct Coulomb ion-ion interactions is $q_{x} q_{y} /\left|\mathbf{R}-\mathbf{R}^{\prime}\right|$ combined with the corresponding contribution from the interaction between the ions and the homogeneous compensating charge. The second term is exactly Eq. (12).

The first term in Eq. (17) is usually associated with the configurational contribution to the energy of the system, but this is correct only if the contribution from intrasite interactions is zero. It is easily evaluated in a completely random alloy, where all the occupation numbers are uncorrelated and therefore $c_{q} c_{q}^{*}=c(1-c) / N$, i.e., normalized to give the ordering energy per atom. One finds

$$
\begin{aligned}
\int_{B Z} d q V(q) c_{q} c_{q}^{*} & =c(1-c) \int_{B Z} d q V(q) \\
& =\Omega_{B Z} c(1-c) V_{R=0},
\end{aligned}
$$

which according to Eq. (13) is equal to $c \epsilon_{0}^{A}+(1-c) \epsilon_{0}^{B}$.

It now remains to define the on-site interaction term $\epsilon_{0}^{i}$, which results from the interaction of the net charge $q_{i}$ in the alloy with the corresponding screening charge, in such a way that $\epsilon_{0}^{i}$ and the corresponding on-site Coulomb potential $V_{i}$ given by Eq. (7) are consistent within DFT, i.e., $V_{i}$ $=\delta \epsilon_{0}^{i} / \delta q_{i}$ :

$$
\epsilon_{0}^{i}=-\frac{e^{2}}{2} \frac{\alpha_{s c r}}{S} q_{i}^{2} .
$$

Using this definition the first term in the Hamiltonian (17) may be written

$$
\begin{aligned}
E_{M a d}^{r a n d-s s}=E_{M a d}^{s c r-s s} & =-\frac{e^{2}}{2} \frac{\alpha_{s c r}}{S}\left[c q_{A}^{2}+(1-c) q_{B}^{2}\right] \\
& =-\frac{e^{2}}{2} c(1-c) \frac{\alpha_{s c r}}{S}\left(q_{A}-q_{B}\right)^{2} \\
& \equiv c(1-c) V_{s c r}(R=0) .
\end{aligned}
$$

This is the result obtained by Magri et al. ${ }^{10}$ more than a decade ago in the so-called charge-correlated model and later by Korzhavyi et al. ${ }^{15,11}$ and by Johnson and Pinski ${ }^{12}$ in their "screened" models for the single-site CPA-DFT. The difference between these models lies only in the way the parameter $\alpha_{\text {scr }}$ is determined (from 0.4397212 for bcc in Ref. 12 to 0.54282038 for fcc in Ref. 20). A discussion of the issues involved may be found in Refs. 12, 20, 23, 24 and 31.

In the supercell aproach, of course, only intersite Coulomb interactions of the net charges contribute to the Madelung energy. The existence of a nonzero Madelung energy in that case is a consequence of the dispersion in the net charges of the $A$ and $B$ components due to different local environment at every site. The difference in the net charges is the result of the fact that the screening charge density goes beond the atomic spheres of the alloy components and therefore sites with different local environments will have a different amount of screening charge. To include the dispersion of charges which exist in the supercell model, one must reformulate the single-site effective-medium model by adding the corresponding on-site screening contribution to the usual intersite part of the Hamiltonian.

\section{B. Configurational part of the Madelung energy and potential in the effective-medium approach}

The reason why we reformulated the Hamiltonian in terms of concentration waves is to show how to define correctly the configurational part of the energy, and in particular the Madelung energy in the presence of on-site interactions. This is an important issue since in some formalisms, such as pseudopotential theory or the $S^{(2)}$ formalism, ${ }^{42-44} V(q)$ is already given a particular form and this may lead to problems with the correct definition of the configurational part of the total energy. The point is that the intrasite interactions do not contribute to the configurational part of the total energy which in real space may be written as (here, we do not consider the contribution from multisite interactions $)^{45}$

$$
H_{\text {conf }}=\frac{1}{2} \sum_{R \neq R^{\prime}} V_{R R^{\prime}} \delta c_{R} \delta c_{R^{\prime}} .
$$

Therefore if the configurational Hamiltonian is written in terms of $V(q)$, e.g., in the concentration wave formalism, it must be corrected by the subtraction of the corresponding intrasite interaction, i.e.,

$$
\begin{aligned}
H_{c o n f} & =\frac{1}{2 \Omega_{B Z}} \int_{B Z} d q V(q) c_{q} c_{q}^{*}-\frac{1}{2} c(1-c) V_{R=0} \\
& =\frac{1}{2 \Omega_{B Z}} \int_{B Z} d q\left[V(q)-V_{R=0}\right] c_{q} c_{q}^{*},
\end{aligned}
$$


where we have used the sum rule for the concentration wave density $c_{q} c_{q}^{*}: \int_{B Z} d q V(q) c_{q} c_{q}^{*}=\Omega_{B Z} c(1-c)$.

The subtraction of the intrasite term in Eq. (22) is crucial for obtaining the correct ordering energy in pseudopotential theory and in the $S^{(2)}$ formalism ${ }^{42-44}$ as well as for making the theory consistent. Let us, for instance, consider the Madelung energy of a binary, completely ordered alloy with two nonequivalent sublattices. It is easy to show that its Madelung energy may be presented in a form similar to that of the Madelung energy of the random alloy. For instance, the Madelung energy of the $L 1_{0}$ ordered phase is

$$
E_{\text {Mad }}^{L 1_{0}}=-\frac{e^{2}}{2} \frac{\alpha_{L 1_{0}}}{S} c(1-c)\left(q_{A}-q_{B}\right)^{2}+\frac{e^{2}}{2} \frac{\alpha_{f c c}}{S} \tilde{q}^{2},
$$

where the last term is zero in the ASA since $\tilde{q}=0$ as in the random alloy case, but now $\alpha_{L 1_{0}}$ is a constant which appears due to the intersite Coulomb interactions. In the appendix of Ref. 37 it is shown that, in fact, $\alpha_{L 1_{0}}\left(q_{A}-q_{B}\right)^{2} / S$ is the Fourier transform of the effective direct electrostatic interaction at the corresponding superstructure vector $k_{L 1_{0}}$ $=2 \pi / a(100)$, i.e., $V_{e s}\left(k_{L 1_{0}}\right)=\alpha_{L 1_{0}}\left(q_{A}-q_{B}\right)^{2} / S$.

On the other hand, $E_{\text {Mad }}^{L 1_{0}}$ may also be found as the sum of the electrostatic energy of the completely random alloy, $E_{\text {Mad }}^{r a n d-s s}$, given by Eq. (20) and the ordering energy, $\Delta U$ : $E_{M a d}^{L 1}=E_{M a d}^{r a n d-s s}+\Delta U$. Since the Madelung energy of the ordered $L 1_{0}$ alloy, $E_{\text {Mad }}^{L 1_{0}}$ is uniquely defined in terms of the corresponding Madelung constant, which has nothing to do with the screening in the alloy, it is obvious that a screening term must be present in the ordering energy $\Delta U$ to compensate for the screening contribution to $E_{\text {Mad }}^{\text {rand }-s s}$.

Indeed, as shown in the appendix of Ref. 45 the ordering energy in the $L 1_{0}$ structure may be written in the form

$$
\Delta U_{M a d}=\frac{1}{8} \eta^{2}\left[V_{e s}\left(k_{L 1_{0}}\right)-V_{s c r}(R=0)\right],
$$

from which it is easy to see that in the completely ordered state, where the long-range order parameter $\eta=1$, the last term in Eq. (24) is exactly the Madelung energy of a random alloy at the stoichiometric composition $[c(1-c)=1 / 4]$, and thus $E_{\text {Mad }}^{\text {rand }-s s}+\Delta U=1 / 8 \alpha_{L 1_{0}}\left(q_{A}-q_{B}\right)^{2} / S$.

This illustrates an important point: The ordering energy represented in reciprocal space in the concentration wave formalism must be corrected by the subtraction of the intrasite term, otherwise the theory will not be consistent. Equation (22) gives the correct definition of the ordering energy considered more thoroughly in the appendix of Ref. 45. The intrasite interaction must also be subtracted when one considers the energy of short-range order (SRO) effects, and thus the correct Krivoglaz-Clapp-Moss expression must have $V(q)-V_{R=0}$ instead of $V(q)$, which is exactly the case in Krivoglaz's derivation. ${ }^{46}$ Note, however, that this problem does not exist if the Krivoglaz-Clapp-Moss expression is used together with the so-called Onsager correction ${ }^{43}$ provided that it is properly defined.

\section{Effective-medium approach to the Madelung potential and energy of a random alloy resented by a supercell}

The reason that it was possible to calculate $\alpha_{s c r}$ on the basis of the ordered structures is the fact that in an ordered binary alloy with only two nonequivalent sublattices one has an exact cancellation of the screening contribution to the Madelung energy and potential. This does not happen, however, in the general case of a supercell with $n>2$ nonequivalent sublattices. For $n=2$, the Madelung energy in the effective-medium approach may be written as the sum of the contribution from the intrasite screening interactions,

$$
\begin{aligned}
E_{\text {Mad }}^{s c r-s c}= & \frac{e^{2}}{2 N} \frac{\alpha_{s c r}}{S} \sum_{i} q_{i}^{2} \\
= & \frac{e^{2}}{2} \frac{\alpha_{s c r}}{S}\left(c \frac{1}{N_{A}} \sum_{i=A} q_{i A}^{2}+(1-c) \frac{1}{N_{B}} \sum_{i=B} q_{i B}^{2}\right) \\
& \times\left(\neq \frac{e^{2}}{2} \frac{\alpha_{s c r}}{S}\left[c\left\langle q_{A}\right\rangle^{2}+(1-c)\left\langle q_{B}\right\rangle^{2}\right]\right),
\end{aligned}
$$

where $N_{A}$ and $N_{B}$ are the number of $A$ and $B$ atoms, respectively, and the ordering energy due to the intersite interactions

$$
\Delta U_{M a d}=\frac{e^{2}}{2 S} \sum_{i} \gamma_{i}\left(\alpha_{k_{i}}-\alpha_{s c r}\right) \Delta q_{k_{i}}^{2}
$$

Here, $\gamma_{i}$ is a normalizing coefficient, $\alpha_{k_{i}}$ a constant due to the bare electrostatic interactions between the net charges for the superstructure vector $k_{i}$ which may be calculated from the Madelung constants $\alpha_{M}^{i j}$ of the corresponding supercell similar to the $\alpha_{L 1}$ considered above, and $\Delta q_{k_{i}}$ the difference between the charges in the crests and in the troughs of the concentration wave in the supercell. In the case of a binary alloy with two nonequivalent sublattices, there is only one $k_{i}$ and $\Delta q_{k_{i}}=\left(q_{A}-q_{B}\right)$.

If $\alpha_{k_{i}}$ depends only on the structure and describes the bare electrostatic interaction between net charges, then $\Delta q_{k_{i}}$ "dresses" these interactions according to the real charge distribution in the alloy (an equivalent description in real space in the charge correlated model is given by Wolverton and Zunger, ${ }^{47}$ who also show that the Madelung energy of the random alloy has intrasite character). If the net charges in the supercell are screened (or uncorrelated) at distances less than half the period of the concentration wave with wave vector $k_{i}$ then $\Delta q_{k_{i}}=0$ due to the destruction interference and the corresponding contribution to the ordering energy vanishes. If the supercell includes only long-range concentration waves, the corresponding ordering contribution to the Madelung potential and energy becomes zero.

Let us finally mention the fact that the Madelung energy of a random alloy obtained in supercell calculations (25) is not equal to the Madelung energy in the single-site calculations, and thus it cannot be used to obtain $\alpha_{s c r}$. The reason is simply that the Madelung energy is not a self-averaging quantity. However, the Madelung potential is, and it is clear that 


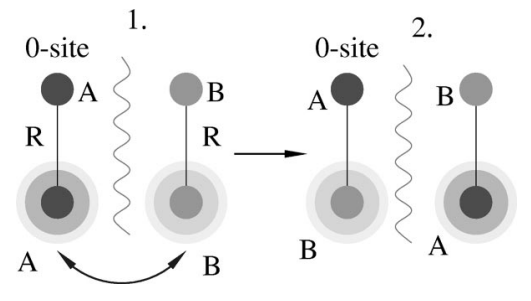

FIG. 7. Two systems, whose 0-site projected Coulomb energy is to be used in the calculation of the screened effective interactions at distance $R$.

$$
\left\langle V_{X}\right\rangle^{s c}=\frac{e^{2}}{N_{X}} \frac{\alpha_{s c r}}{S} \sum_{i=x} q_{i X}=e^{2} \frac{\alpha_{s c r}}{S}\left\langle q_{X}\right\rangle=V_{X}^{s s},
$$

which allows one to use Eq. (8) to obtain $\alpha_{s c r}$ in the supercell calculations and shows why $\alpha_{\text {rand }}$ is exactly equal to $\alpha_{s c r}$.

\section{Intersite screened Coulomb interactions}

Although the screened Coulomb interactions have an intrasite character, they may contribute to the effective pair intersite interactions of the kind obtained in the generalized perturbation method (GPM), ${ }^{19,48}$ because the screening charge is located on several of the coordination shells around each atom. This was, in fact, already recognized by Ducastelle $^{19}$ who derived the contribution to the GPM potentials from the screened Coulomb interactions in the framework of the Hartree-Fock tight-binding CPA theory.

The existence of an additional electrostatic term due to the screening is also consistent with Andersen's force theorem, ${ }^{49}$ which states that the change in the total energy of a system due to some perturbation to first order is given by the change in the sum of the one-electron energies obtained from frozen one-electron potentials plus the change of the electrostatic energy due to the perturbation. In fact, this latter contribution from the screened Coulomb interaction has been completely neglected in a number of first-principles calculations of GPM interactions. ${ }^{50-53}$ Here we will therefore show how the screening contribution to the GPM potentials may be defined and obtained on the basis of the calculated spatial distribution of the screening charge.

GPM-like pair interactions, usually defined by Eq. (16) for a specific lattice vector $\mathbf{R}$, may be determined as the site-projected part of the change in the total energy when two atoms of different types in a completely random alloy are exchanged between sites infinitely far apart in such a way that their neighbors at the relative position $\mathbf{R}$ are of the opposite type after the exchange. This is schematically illustrated in Fig. 7. That part of the total energy which should be accounted for is half the site-decomposed total energy written in terms of the intersite interactions or interatomic potentials, i.e.,

$$
V^{(2)}(R)=\frac{1}{2}\left[E_{1}^{(2)}(R)-E_{2}^{(2)}(R)\right]
$$

Here, $E_{1}^{(1)}$ is the total energy due to pairwise interactions of the unperturbed system projected onto site 0 and $E_{2}^{(2)}$ is the same quantity after the exchange. A similar expression is also valid in the case of multisite interactions, but this will involve a more complex exchange of atoms and will not be considered here because the screened Coulomb interactions do not contribute to the effective multisite interactions in the ASA.

Within multiple-scattering theory as well as in the tightbinding approximation a Green's-function formulation allows both site and "path" decomposition of the electron density and thereby makes it possible to write down an analytical expression for the one-electron contribution to the $n$-site interactions, $V^{(n)}(R)$, in the CPA. ${ }^{19,48}$ Concerning the screened Coulomb interactions one must, however, proceed differently. There are several ways to do so, but here we will present a straightforward approach.

In the sense of the CPA and single-site mean-field theory we will use an effective-medium approach, assuming that at all sites, i.e., within the atomic spheres assigned to each site, there is an electroneutral effective medium except at the two sites $\mathbf{0}$ and $\mathbf{R}$ under consideration. In those two sites we must use the actual values of the net charges of the alloy components, which in the effective-medium approach are the average net charges $q_{A}$ and $q_{B}$ of the alloy components.

In the first-principles methods, however, these net charges depend on the specific choice of the size of the atomic spheres and thus they must, in principle, go together with the corresponding screening cloud. Since we calculate the change in the electrostatic energy of the two systems shown in Fig. 7 projected onto site $\mathbf{0}$ due to the exchange of $A$ and $B$ atoms in positions $\mathbf{R}$, we must include only the interaction of the net charge at site $\mathbf{0}$ with the net charge at site $\mathbf{R}$ and its screening charge. That is, the interaction of the net charge with its own screening charge must be excluded as it is included in the definition of the screened on-site interactions; see Eq. (11). Thus the first term in Eq. (28) for the system before the exchange of atoms has been made, $E_{1}^{s c r}(R)$, is

$$
E_{1}^{s c r}(R)=e^{2} q_{A} \sum_{\mathbf{R}^{\prime} \neq 0} \frac{q_{A \mathbf{R}^{\prime}}}{R^{\prime}}+e^{2} q_{B} \sum_{\mathbf{R}^{\prime} \neq 0} \frac{q_{B} \mathbf{R}^{\prime}}{R^{\prime}} .
$$

Here, $q_{i \mathbf{R}^{\prime}}$ is either the net charge of the $i$ th component $q_{i}$ if $\mathbf{R}^{\prime}=\mathbf{R}$ or the corresponding screening charge if $\mathbf{R}^{\prime} \neq \mathbf{R}$. A similar expression may be written for $E_{2}^{s c r}(R)$, after the exchange of the $A$ and $B$ atoms in the $\mathbf{R}$ sites, i.e.,

$$
E_{2}^{s c r}(R)=e^{2} q_{A} \sum_{\mathbf{R}^{\prime} \neq 0} \frac{q_{B} \mathbf{R}^{\prime}}{R^{\prime}}+e^{2} q_{B} \sum_{\mathbf{R}^{\prime} \neq 0} \frac{q_{A \mathbf{R}^{\prime}}}{R^{\prime}} .
$$

The resulting expression for the screened Coulomb interactions which should be added to the usual one-electron term is therefore

$$
\begin{aligned}
V_{s c r}(R) & =\frac{e^{2}}{2}\left(q_{A} \sum_{\mathbf{R}^{\prime} \neq 0} \frac{q_{A \mathbf{R}^{\prime}}-q_{B \mathbf{R}^{\prime}}}{R^{\prime}}-q_{B} \sum_{\mathbf{R}^{\prime} \neq 0} \frac{q_{B \mathbf{R}^{\prime}}-q_{A \mathbf{R}^{\prime}}}{R^{\prime}}\right) \\
& =\frac{e^{2}}{2}\left(q_{A}-q_{B}\right)^{2} \sum_{\mathbf{R}^{\prime} \neq 0} \frac{Q\left(\left|\mathbf{R}^{\prime}-\mathbf{R}\right|\right)}{R^{\prime}}
\end{aligned}
$$




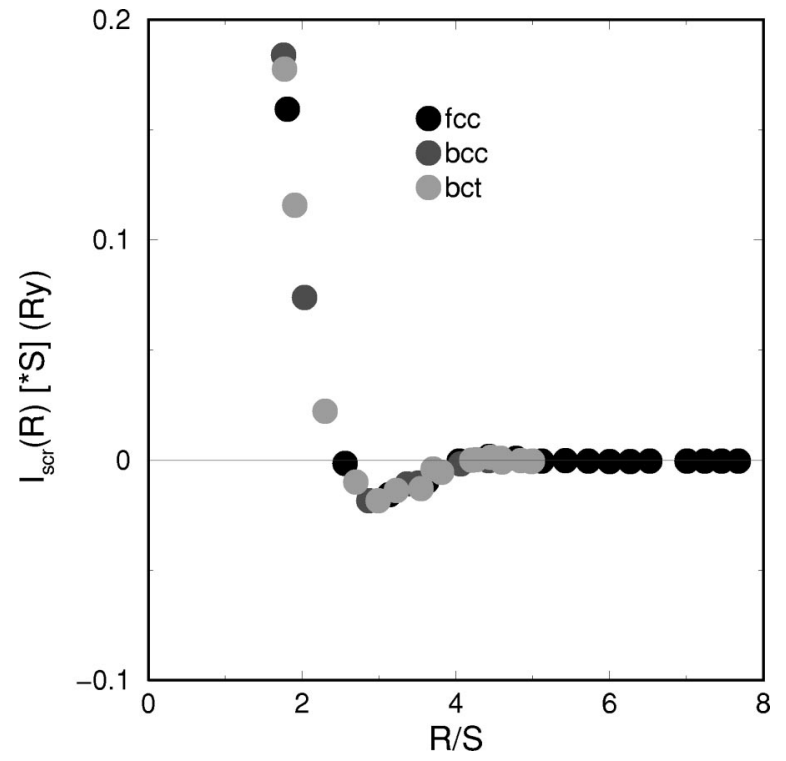

FIG. 8. The intersite screened Coulomb effective interactions obtained from the normalized screening charge presented in Fig. 6.

where $Q(R)$ is the normalized screening charge defined in Eq. (9), and where we have used the condition that the screening does not depend on the type of the atom. Finally, performing the summation in Eq. (31) one may define the screened Coulomb interactions as

$$
V_{s c r}(R)=\frac{e^{2}}{2}\left(q_{A}-q_{B}\right)^{2} \frac{\alpha_{s c r}(R)}{S} .
$$

It is easy to see from Eqs. (31) and (11) that $\alpha_{s c r}(R=0)$ $=\alpha_{s c r}=\alpha_{\text {rand }}$ and therefore $V_{s c r}(R=0)$ is exactly the onsite screened interactions that defines the Madelung energy of the binary alloy which has exactly the same form (20). This on-site interaction must be included in the definition of the $S^{(2)}$ interactions, ${ }^{42}$ as has been demonstrated in the previous section (see also Ref. 44). When $R \neq 0, V_{s c r}(R)$ defines the intersite screened Coulomb interaction contribution to the GPM-like effective interactions. Since the screening in the ASA is practically universal these interactions have the universal form presented in Fig. 8.

\section{TOTAL ENERGY IN THE SINGLE-SITE CPA AND THE SUPERCELL LSGF METHODS}

The fact that the Madelung energy of a random alloy described either by the effective-medium model defined by the SS-DFT-CPA method or by the supercell model in conjunction with the SS-LSGF method differ from each other has neither consequences for the final result for the total energy of the random alloy nor even for the partial and local contributions to the total energy. This follows simply from the fact that the density of states and its average local contributions are the same in the two methods, as shown above.

In Table I we compare the total energy and its components in a $\mathrm{Cu}_{50} \mathrm{Pt}_{50}$ random alloy calculated by the SS-DFT-CPA method with $\alpha_{s c r}=0.60572$ and by the SS-LSGF method on the basis of a 512-atom supercell, in which the atomic positions of $\mathrm{Cu}$ and $\mathrm{Pt}$ have been chosen such that the SRO parameters are equal to zero at the first seven coordination

TABLE I. The total energy, (in Ry) of $\mathrm{Cu}_{50} \mathrm{Pt}_{50}$ random alloy and corresponding contributions obtained in three different calculations: by the single-site CPA-DFT method, (ss-CPA-DFT), in the 512-atom supercell LSGF calculations with optimized atomic distribution, providing zero SRO parameters up to the seventh coordination shell, (LSGF-1), and with atomic configuration immediately after random number generator, (LSGF-2)., $\left(E_{\text {coul }}=E_{e l-n u c}+E_{e l-e l}+E_{\text {Mad }}\right)$.

\begin{tabular}{|c|c|c|c|c|}
\hline Site & Energy & ss-CPA-DFT & LSGF-1 & LSGF-2 \\
\hline \multicolumn{5}{|l|}{$\mathrm{Cu}$} \\
\hline & Kinetic & 3360.076110 & 3360.076294 & 3360.077674 \\
\hline & $\left\langle E_{e l-n u c}\right\rangle$ & -7974.160832 & -7974.157257 & -7974.178557 \\
\hline & $\left\langle E_{e l-e l}\right\rangle$ & 1439.080777 & 1439.078359 & 1439.099272 \\
\hline & $\left\langle E_{M a d}\right\rangle$ & -0.004193 & -0.005646 & -0.005994 \\
\hline & $\left\langle E_{\text {Coul }}\right\rangle$ & -6535.084248 & -6535.084544 & -6535.085279 \\
\hline & $\left\langle E_{x c}\right\rangle$ & -130.026085 & -130.026000 & -130.026621 \\
\hline & $\left\langle E_{C u}\right\rangle$ & -3305.034222 & -3305.034250 & -3305.034226 \\
\hline \multicolumn{5}{|l|}{$\mathrm{Pt}$} \\
\hline & Kinetic & 42188.794140 & 42188.794273 & 42188.791806 \\
\hline & $\left\langle E_{e l-n u c}\right\rangle$ & -92747.101049 & -92747.093691 & -92747.030284 \\
\hline & $\left\langle E_{e l-e l}\right\rangle$ & 14378.917863 & 14378.911671 & 14378.849707 \\
\hline & $\left\langle E_{M a d}\right\rangle$ & -0.004193 & -0.005533 & -0.005472 \\
\hline & $\left\langle E_{\text {Coul }}\right\rangle$ & -78368.187379 & -78368.187553 & -78368.186049 \\
\hline & $\left\langle E_{x c}\right\rangle$ & -693.866856 & -693.866786 & -693.865919 \\
\hline & $\left\langle E_{P t}\right\rangle$ & -36873.260095 & -36873.260068 & -36873.260162 \\
\hline Alloy & $E_{t o t}$ & -20089.147159 & -20089.147159 & -20089.147194 \\
\hline
\end{tabular}


shells (LSGF-1). The agreement between the two calculations is seen to be excellent if one combines the electronnucleus, the electron-electron, and the Madelung contributions to form a total Coulomb energy, $E_{\text {coul }}=E_{\text {el-nuc }}$ $+E_{e l-e l}+E_{\text {Mad }}$.

The accuracy of the SS-DFT-CPA method with the appropriate screening contribution to the Madelung potential and energy may be appreciated if one compares the results of a 512-atom supercell calculation performed by the SS-LSGF method (LSGF-2) where the distribution of the $\mathrm{Cu}$ and $\mathrm{Pt}$ atoms have not been optimized after the application of the random number generator leading to quite small, but not zero, SRO parameters. The values of the SRO parameters for the first seven coordination shells are -0.005208 (1), 0.026041 (2), 0.007161 (3), -0.014323 (4), -0.021484 (5), $0.0390625(6),-0.0136718$ (7), respectively, which are approximately the same, as in the LSMS calculations in Ref. 54. The agreement between SS-DFT-CPA results and SSLSGF calculations with a properly chosen supercell (LSGF-1) is obviously better than between two SS-LSGF calculations.

\section{CONCLUSION}

The screened Coulomb interactions which are due to the interaction between the net charge of an alloy component and its screening charge must be included in a consistent single-site mean-field theory of the electrostatics in random alloys. In this paper we have shown how this may be done and we have calculated the spatial distribution of the screening charge which in the ASA is found to be practically universal for homogeneous systems. A formalism that describes the contribution from for the screened Coulomb interaction to Madelung potential and energy as well as to the effective interactions of the GPM-type is presented.

\section{ACKNOWLEDGMENTS}

Valuable discussions with Dr. P. A. Korzhavyi, I. A. Abrikosov, A. Yu. Lozovoi, Professor A. Gonis, and Professor S. Faulkner are greatly acknowledged. The center for AtomicScale Materials Physics is sponsored by the Danish National Research Foundation.
${ }^{1}$ P. Soven, Phys. Rev. 156, 809 (1967).

${ }^{2}$ D.W. Taylor, Phys. Rev. 156, 1017 (1967).

${ }^{3}$ S. Kirkpatrick, B. Velicky, and H. Erenreich, Phys. Rev. B 1, 3250 (1970).

${ }^{4}$ B.L. Gyorffy and G.M. Stocks, in Electrons in Disordered Metals and at Metallic Surfaces, edited by P. Phariseau, B. L. Gyorffy, and L. Scheire (Plenum, New York, 1978).

${ }^{5}$ J.S. Faulkner, Prog. Mater. Sci. 27, 1 (1982).

${ }^{6}$ P. Hohenberg and W. Kohn, Phys. Rev. 136, B864 (1964).

${ }^{7}$ W. Kohn and L.J. Sham, Phys. Rev. 140, A1133 (1965).

${ }^{8}$ D.D. Johnson, D.M. Nicholson, F.J. Pinski, B.L. Gyorffy, and G.M. Stocks, Phys. Rev. Lett. 56, 2088 (1986).

${ }^{9}$ D.D. Johnson, D.M. Nicholson, F.J. Pinski, B.L. Gyorffy, and G.M. Stocks, Phys. Rev. B 41, 9701 (1990).

${ }^{10}$ R. Magri, S.-H. Wei, and A. Zunger, Phys. Rev. B 42, 11388 (1990).

${ }^{11}$ I.A. Abrikosov, Yu.H. Vekilov, P.A. Korzhavyi, A.V. Ruban, and L.E. Shilkrot, Solid State Commun. 83, 867 (1992).

${ }^{12}$ D.D. Johnson and F.J. Pinski, Phys. Rev. B 48, 11553 (1993).

${ }^{13}$ P.P. Singh, A. Gonis, and P.E.A. Turchi, Phys. Rev. Lett. 71, 1605 (1993).

${ }^{14}$ I.A. Abrikosov, A.M.N. Niklasson, S.I. Simak, B. Johansson, A.V. Ruban, and H.L. Skriver, Phys. Rev. Lett. 76, 4203 (1996).

${ }^{15}$ P.A. Korzhavyi (unpublished).

${ }^{16}$ R. Zeller, J. Phys. F: Met. Phys. 17, 2123 (1987).

${ }^{17}$ N. Stefanou, A. Oswald, R. Zeller, and P.H. Dederichs, Phys. Rev. B 35, 6911 (1987).

${ }^{18}$ G. Treglia, F. Ducastelle, and F. Gautier, J. Phys. F: Met. Phys. 8, 1437 (1978).

${ }^{19}$ F. Ducastelle, Order and Phase Stability in Alloys (NorthHolland, Amsterdam, 1991).

${ }^{20}$ I.A. Abrikosov and B. Johansson, Phys. Rev. B 57, 14164 (1998).

${ }^{21}$ A. Gonis, P.E. Turchi, J. Kudrnovsky, V. Drchal, and I. Turek, J. Phys.: Condens. Matter 8, 7883 (1996).
${ }^{22}$ B. Ujfalussy, J.S. Faulkner, N.Y. Moghadam, G.M. Stocks, and Y. Wang, Phys. Rev. B 61, 12005 (2000).

${ }^{23}$ P.A. Korzhavyi, A.V. Ruban, I.A. Abrikosov, and H.L. Skriver, Phys. Rev. B 51, 5773 (1995).

${ }^{24}$ A.V. Ruban, I.A. Abrikosov, and H.L. Skriver, Phys. Rev. B 51, 12958 (1995).

${ }^{25}$ J.S. Faulkner, Yang Wang, and G.M. Stocks, Phys. Rev. B 52, 17106 (1995).

${ }^{26}$ I.M. Lifshitz, S.A. Gredeskul, and L.A. Pastur, Introduction to The Theory of Disordered Systems (John Wiley, New York, 1987).

${ }^{27}$ J.M. Sanchez, F. Ducastelle, and D. Gratias, Physica A 128, 334 (1984).

${ }^{28}$ A. Zunger, S.-H. Wei, L.G. Ferreira, and J.E. Bernard, Phys. Rev. Lett. 65, 353 (1990).

${ }^{29}$ I.A. Abrikosov, S.I. Simak, B. Johansson, A.V. Ruban, and H.L. Skriver, Phys. Rev. B 56, 9319 (1997).

${ }^{30}$ A. Gonis and J.W. Garland, Phys. Rev. B 16, 2424 (1977); C.W. Myles and J.D. Dow, Phys. Rev. Lett. 42, 254 (1979); A. Gonis, W.H. Butler, and G.M. Stocks, ibid. 50, 1482 (1982); A. Gonis, G.M. Stocks, W.H. Butler, and H. Winter, Phys. Rev. B 29, 555 (1984).

${ }^{31}$ C. Wolverton, A. Zunger, S. Froyen, and S.-H. Wei, Phys. Rev. B 54, 7843 (1996).

${ }^{32}$ A.V. Ruban and H.L. Skriver, Comput. Mater. Sci. 15, 119 (1999).

${ }^{33}$ J.P. Perdew and A. Zunger, Phys. Rev. B 23, 5048 (1981).

${ }^{34}$ D.M. Ceperley and B.J. Alder, Phys. Rev. Lett. 45, 566 (1980).

${ }^{35}$ J.S. Faulkner, N.Y. Moghadam, Y. Wang, and G.M. Stocks, Phys. Rev. B 57, 7653 (1998).

${ }^{36}$ F.J. Pinski, Phys. Rev. B 57, 15140 (1998).

${ }^{37}$ A.I. Landa, A.A. Yuryev, A.V. Ruban, E.G. Gurskaya, Yu.K. Kovneristyi, and N.A. Vatolin, J. Phys.: Condens. Matter 3, 9229 (1991). 
${ }^{38}$ R.E. Watson, M. Weinert, and G.W. Fernando, Phys. Rev. B 43, 1446 (1991).

${ }^{39}$ F.W. Averill and G.S. Painter, Phys. Rev. B 41, 10344 (1990).

${ }^{40}$ B. Drittler, M. Weinert, R. Zeller, and P.H. Dederichs, Phys. Rev. B 39, 930 (1989).

${ }^{41}$ G.L. Krasko, JETP Lett. 13, 218 (1971).

${ }^{42}$ B.L. Gyorffy and G.M. Stocks, Phys. Rev. Lett. 50, 374 (1983).

${ }^{43}$ J.B. Staunton, D.D. Johnson, and F.J. Pinski, Phys. Rev. B 50, 1450 (1994).

${ }^{44}$ F.J. Pinski, J.B. Staunton, and D.D. Johnson, Phys. Rev. B 57, 15177 (1998).

${ }^{45}$ A.V. Ruban and H.L. Skriver, Phys. Rev. B 55, 856 (1997).

${ }^{46}$ M.A. Krivoglaz and A.A. Smirnov, The Theory of Order-Disorder in Alloys (McDonald, London, 1964).

${ }^{47}$ C. Wolverton and A. Zunger, Phys. Rev. B 51, 6876 (1995).
${ }^{48}$ F. Ducastelle and F. Gautier, J. Phys. F: Met. Phys. 6, 2039 (1976).

${ }^{49}$ A.R. Mackintosh and O.K. Andersen, in Electrons at the Fermi Surface, edited by M. Springford (Cambridge University Press, Cambridge, England, 1980).

${ }^{50}$ A. Gonis, X.-G. Zhang, A.J. Freeman, P. Turchi, G.M. Stocks, and D.M. Nicholson, Phys. Rev. B 36, 4630 (1987).

${ }^{51}$ P.E.A. Turchi, G.M. Stocks, W.H. Butler, D.M. Nicholson, and A. Gonis, Phys. Rev. B B37, 5982 (1988).

${ }^{52}$ V. Drchal, J. Kudrnovský, L. Udvardi, P. Weinberger, and A. Pasturel, Phys. Rev. B 45, 14328 (1992).

${ }^{53}$ P.P. Singh and A. Gonis, Phys. Rev. B 47, 6744 (1993).

${ }^{54}$ J.S. Faulkner, Y. Wang, and G.M. Stocks, Phys. Rev. B 55, 7492 (1997). 